Main Outcome Measurements Prevalence and characteristics of LBP and C-LBP and their determinants.

Results LBP had a prevalence of $91,6 \%$ and $74,2 \%$ for lifetime and 1-year respectively, while C-LBP had a prevalence of $23,9 \%$. Prevalence of LBP in Show-Jumping was $61 \%$, in Dressage 13,6\%, in Eventing 6,3\%, in Horse Country Riding 2,9\% and in Reining 2,2\%. Weight has a positive association with LBP (O.R. $1.05 \quad 95 \% \quad \mathrm{CI}=[1.02,1.09]$ $\mathrm{p}<0.05$ ), while age has a negative association (O.R. 0.95 $95 \% \mathrm{CI}=[0.94,0.98] \mathrm{p}<0.05)$. Practicing activity more than 5-6 hours a week has a positive association with CLBP (O.R. $1.2195 \% \quad \mathrm{CI}=[1.05,1.4] \mathrm{p}<0.05)$. C-LBP is associated with interrupted activity $(\mathrm{p}<0.001)$, drugs consumption $(\mathrm{p}<0.001)$ and restriction in participation $(\mathrm{p}<$ 0.001).

Conclusions LBP and C-LBP are very common conditions in equestrian athletes and their prevalence is higher compared to general population and other sports. LBP is more frequent in show-jumping compared to other disciplines. Age and weight are associated with lifetime LBP, with, respectively, a negative and positive association. Athletes with C-LBP showed more disability in activities of daily living (ADLs) and tendency to suspend sports more frequently.

\section{NEUROMUSCULAR RESPONSES OF THE HAMSTRING AND TRUNK MUSCLES DURING UNANTICIPATED TRUNK PERTURBATIONS}

${ }^{1}$ Ayako Higashihara, ${ }^{2} J u r d a n$ Mendiguchia, ${ }^{3}$ Takashi Ono, ${ }^{4}$ Yasuharu Nagano ${ }^{5}$ Shogo Sasaki, ${ }^{6,7}$ Shinshiro Mineta, ${ }^{8}$ Norikazu Hirose. ${ }^{1}$ Institute of Physical Education, Keio University, Kanagawa, Japan; ${ }^{2}$ Department of Physical Therapy, Zentrum Rehabilitation and Performance Center, Pamplona, Spain; ${ }^{3}$ Kitasato University College of Liberal Arts and Sciences, Kanagawa, Japan; ${ }^{4}$ Japan Women's College of Physical Education, Department of Sports Wellness Sciences, Tokyo, Japan; ${ }^{5}$ Faculty of Health Sciences, Tokyo Ariake University of Medical and Health Sciences, Tokyo, Japan; ${ }^{6}$ Graduate school of Sport and Exercise Sciences, Osaka University of Health and Sport Sciences, Osaka, Japan; ${ }^{7}$ The Japan Society for the Promotion of Science, Tokyo, Japan; ${ }^{8}$ Faculty of Sport Sciences, Waseda University, Saitama, Japan

10.1136/bjsports-2021-IOC.123

Background Trunk movement is considered to be involved in lower extremity injuries. Hamstring strain injuries often occur when movements are unanticipatedly perturbed by the opponents.

Objective To examine the neuromuscular responses of the hamstring and trunk muscles during unanticipated trunk perturbations in the athletes with and without a history of hamstring strain injury.

Design Descriptive laboratory study.

Setting College athletes.

Participants Male college athletes were recruited, 11 with a history of unilateral hamstring strain injury and 10 without prior injury.

Assessment In the kneeling position, the participants wore a chest harness attached to a cable that was pulled backward as a resisting force. They were instructed to resist the force isometrically and keep their initial position as possible as they could when the perturbations were applied. The force was released with a cue (CUE) and without cue (NoCUE). Trunk acceleration, three-dimensional kinematic data, and surface electromyography (EMG) signals of the erector spinae, internal oblique, gluteus maximus, biceps femoris, and semitendinosus muscles were measured.
Main Outcome Measurements (1) Maximum trunk acceleration; (2) angular displacement of the trunk, pelvis, hip, and knee; (3) onset latency; (4) EMG activation in the 50-ms window before the perturbation; and (5) EMG activation in the $100 \mathrm{~ms}$ after the perturbation.

Results The maximum trunk acceleration and displacement were significantly greater during the NoCUE than during the CUE in both groups $(p<0.05)$. The injured group demonstrated significantly delayed onset of the gluteus maximus and erector spinae muscles during the NoCUE compared with the CUE $(p<0.05)$, while no difference was observed in the uninjured group. There was no difference in the phasic EMG activities between groups.

Conclusions Athletes with a history of hamstring strain injury displayed reduction in the neuromuscular coordination of pelvis and trunk muscles when they controlled the unanticipated trunk movement.

\section{MODIFIED KETTLEBELL SQUATS COULD BE SAFER FOR THE LOW BACK OF ATHLETES WITH LIMITED ANKLE DORSIFLEXION}

Sahin Dogukan Kasapoglu, Gulmez Irfan, Selda Uzun, Ramazanoglu Nusret, Goktug Sanli, Vildan Gozde Gumusbas, Yasar Tatar. Faculty of Sport Science, Marmara University, Istanbul, Turkey

\subsection{6/bjsports-2021-IOC.124}

Background Kettlebell squats, holding the kettlebell in front of body, such as goblet squats (GS) can increase range of squat motion. However, the effects of GS variations on postural balance, hip kinematics and their relationship with underlying postural restrictions are not known.

Objective The purpose of this study was to determine the best kettlebell squat exercise variation for improving the strength and postural balance while preserving anterior pelvic tilt for low back health of the athletes with different dorsiflexion range of motion (DFROM) capacities.

Design Single session repeated - measurement

Setting Sport Health Laboratory.

Participants 32 male athletes $(22,1 \pm 1,8$ years; 177,7 \pm $5,1 \mathrm{~cm} ; 73,3 \pm 5,4 \mathrm{~kg}$; Training years: $4.9 \pm 2.2$ ) were included in this study.

Main Outcome Measurements Athletes performed 8 different squat positions on the force platform. Balance measurements were recorded at $100 \mathrm{~Hz}$ for 30 seconds. Kettlebell equipment which was $8 \mathrm{~kg}$, was held (1) close to the trunk (elbows flexed) (GS-EF) and held away from the trunk (elbow extended) (GS-EE) during the goblet squat. Digital inclinometer was used to measure pelvic tilt angle.

Results Postural balance parameters (COP area and antero-posterior sway) during GS-EF were significantly lower in both squat and split squat positions in the dominant leg ( $p$ $<0.001)$. However, it was found that posterior pelvic tilt was lower with GS-EE compared to GS-EF, which shows that athletes maintained their neutral lordosis better with GS-EE. In addition, the athletes with lower DFROM posterior tilted more with GS-EF ( $p$ <0.01). Posterior pelvic tilt was lower during split GS compared to bilateral GS, meaning athletes stayed closer to their neutral lumbar lordosis during split GS $(\mathrm{p}<0.05)$.

Conclusions Athletes with restricted DFROM could incorprate regular or split GS-EE exercises for minimize the low back injury risk. Additionally, higher postural sway during GS-EE 
may indicate a need for higher activation of core muscles. Therefore, GS-EE exercises could also develop core muscles that have protective mechanism on low back.

\section{WHAT ARE THE MOVEMENT PATTERNS ASSOCIATED WITH GOOD AND POOR LUMBOPELVIC STABILITY?}

${ }^{1}$ Margaret Perrott, ${ }^{1}$ jill Cook, ${ }^{2}$ Don Vicendese, ${ }^{1}$ Tania Pizzari. 'La Trobe Sport and Exercise Medicine Research Centre, Melbourne, Australia; ${ }^{2}$ La Trobe University, Melbourne, Australia

\subsection{6/bjsports-2021-IOC.125}

Background Poor lumbopelvic stability (LPS), defined as the lack of optimal alignment of the spine, pelvis, and thigh, is a risk factor for sports injury. Clinicians can validly assess LPS using rating criteria for two movement tests: single leg squat (SLS) and dip test (DT) to assess movement pattern errors. LPS is typically categorised as good, poor or neither but simplistic categories are not sensitive to change nor provide direction for management. Specific movement errors made in each test may be more sensitive to change after exercise interventions than the three-category rating and require investigation.

Objective To establish which movement errors and demographic factors are associated with LPS categories.

Design Observational study.

Setting Adult recreational athletes.

Participants Recreational athletes ( $\mathrm{n}=122,50$ men, 73 women) 18-49 years, playing land-based sports, with no conditions preventing performance of movement tests.

Assessment of Risk Factors Independent variable: LPS rating category: good, neither, poor.

Main Outcome Measurements Athletes were filmed performing SLS and DT on each leg. Two physiotherapists independently categorised their LPS, noting the presence of movement errors defined in the rating criteria. Dependent variable: movement errors, demographic factors.

Results Good LPS was associated with the absence of specific movement errors: trunk lateral flexion or rotation, hip adduction and jerky movement in SLS and pelvic obliquity and jerky movement in DT (sensitivity 0.97, specificity 0.94). Poor LPS was associated with hip abduction (non-trial leg) in SLS, jerky movement in DT and limited ankle dorsiflexion (sensitivity 0.80 , specificity 0.75$)$. An increase of $2^{\circ}$ dorsiflexion reduced the risk of being categorised as having poor LPS by $26 \%$. Increasing age was associated with sub-optimal LPS ( $p$ $=0.014$ ).

Conclusions Specific movement errors, and limited dorsiflexion and increasing age are associated with sub-optimal LPS. Strength programs improving movement control and mobility exercises improving ankle dorsiflexion should be implemented. As athletes age they should give more attention to maintaining optimal LPS.

\section{PSYCHOSOCIAL FACTORS ARE ASSOCIATED WITH LOWER RE-INJURY RISK IN COMPETITIVE ATHLETES}

Adam Gledhill, Ross Craig. Leeds Beckett University, Leeds, UK

10.1136/bjsports-2021-IOC.126

Background Psychosocial factors have achieved growing acceptance in their role in successful return to sport. However, as yet, few studies have demonstrated which of the commonly cited psychosocial variables can most strongly predict re-injury rates in competitive athletes. Understanding this could support clinicians in best directing valuable resource towards the holistic support of injured athletes, with a view to facilitating a successful return to sport.

Objective To examine whether social support, psychological readiness to return to sport and re-injury anxiety can predict re-injury in competitive athletes

Design Retrospective, cross-sectional study.

Setting Competitive sport.

Participants 141 competitive athletes, from a range of sports, aged 19 to 24 years (mean age: 20.1 years; SD 1.1 years; 72 male and 69 female).

Interventions (or Assessment of Risk Factors) Independent variables injury time-loss, perceived availability of social support, psychological readiness to return to sport and re-injury anxiety.

Main Outcome Measurements Re-injury.

Results There was a significant difference $(p=<0.001)$ between competitive athletes who re-injured the same injury versus those who didn't. Specifically, re-injury anxiety was lower in athletes who did not re-injure, and perceived availability of social support and psychological readiness to return to sport were higher in athletes who did not re-injure. Athletes who did not re-injure also had a longer return to sport. There was no significant difference in injury rates between male and female athletes $(p=.105)$. Regression analysis indicated that $61.9 \%$ of variance in re-injury rates was predicted by the included predictor variables, with the most significant predictors of reduced re-injury risk being perceived availability of informational support $(p=0.003)$ and time out of sport $(p=0.003)$.

Conclusions Clinicians seeking to reduce the risk of re-injury in competitive athletes should consider strategies to reduce reinjury anxiety and facilitate the provision of social support, specifically the provision of high-quality informational support. Delayed return to sport is also important in reducing the risk of re-injury.

\section{PSYCHOLOGICAL DISTRESS AND WELLBEING IN UK OLYMPIC AND PARALYMPIC ATHLETES}

${ }^{1}$ Craig Ranson, ${ }^{2}$ Sandra Leyland, ${ }^{2}$ Lisa Board, ${ }^{1}$ Rod Jaques, 1,2,3 Alan Currie. ${ }^{1}$ English Institute of Sport, Manchester, UK; ${ }^{2}$ University of Sunderland, Sunderland, UK ${ }^{3}$ Northumberland Tyne and Wear NHS Foundation Trust, Newcastle, UK

\subsection{6/bjsports-2021-IOC.127}

Background Despite the known mental health benefits of exercise the prevalence of mental health symptoms and disorders in high performance athletes appears to be slightly higher than in the general population and athletes with disabilities 